\title{
The Analysis of the EU-ETS Carbon Allowance Cap Setting Method
}

\author{
Bin $\mathrm{Ye}^{1}$, Lixin Miao ${ }^{1,}$, Jingjing Jiang ${ }^{2, b}$ and Peng Yang ${ }^{1}$ \\ ${ }^{1}$ Shenzhen Logistics Engineering and Simulation Laboratory, Graduate School at Shenzhen, \\ Tsinghua University, Shenzhen 518055, China \\ ${ }^{2}$ South University of Science and Technology of China, Shenzhen, 518055, China \\ a Ixmiao@tsinghua.edu.cn, b jjjing55@163.com
}

\begin{abstract}
One of the most important works of establishing a carbon trading market is to set the allowance cap. For a long time, no matter in Europe or China, most of experts and professionals have believed the cap of allowance to be fixed. The reason seemed clear enough: Fixed cap can guarantee stable anticipated supply in carbon market, which is the basis of market equilibrium, the necessary condition for enterprises to make consistent decisions, and is beneficial for building up allowance pricing mechanism based on marginal carbon emission reduction cost; therefore it can ensure the effectiveness of carbon market. However, this theoretical assumption deviates from practical result, leading to an antinomy. The theoretical assumption is that fixed cap as the basis of the orderliness of carbon market. The fact is that EU ETS, as a carbon market strictly implementing cap of allowance, not only has violent price fluctuations, but also needs to adjust its cap periodically, which results in another round of disorderly violent fluctuation of allowance price. This paper endeavors to find out the reasons for the paradox by analyzing the theoretical logic of EU-ETS adopting fixed cap.
\end{abstract}

Keywords: EU-ETS; carbon market; cap setting.

\section{Introduction}

Cap-and-trade currently dominates international carbon emission trading systems, and its basic framework includes two parts: first, to set an allowance cap and allocate allowance; second, to establish a trading market, where the covered entities can reduce cost for compliance by trading allowances, to reduce emissions of greenhouse gases through effective market trading. Now it is adopted by EU ETS, RGGI, California ETS, Australian ETS, Tokyo ETS and others, where EU ETS is the most representative and originative [1].

\section{The basic feature of EU-ETS}

The European Union has taken the lead in entering post-industrial stage. Its total carbon emission has reached a peak value and its economy grows stably with an average annual growth rate of 1 to $2 \%$. It has formed an industrial structure dominated by service industry where manufacturing only has a small proportion in the economy and many low-end production chains have been shifted outside. The carbon emission structure of EU has been stabilized: energy processing and conversion is the largest carbon emission sector of EU (38\%), transportation is the second largest (25\%), residence and commerce is the third largest (20\%), and manufacturing and architecture has a small proportion (16\%), forming a carbon emission structure with ratio of 3:2:2:1; however, carbon emission from energy process and conversion, and manufacturing is on the downside while the transportation sector is rapidly increasing. Given the above economic situations and carbon emission features, in 2005, EU launched EU ETS with "cap-and-trade", which can be generally expressed as: first, in accordance with emission reduction targets and economic projections, to set allowance cap for compliance phase and to allocate the allowance; once the cap and its allocation are determined, they will not be adjusted; then to motivate covered entities to realize carbon emission compliance and cost risk controlling by trading allowance with its derivative products, finding market price of allowance resources in the carbon exchange transactions[2].

Since its official launching in 2005, EU ETS has made great achievements and is currently the practice of cap-and-trade that has the largest scale and coverage, which has model effects for carbon emission trading markets worldwide. First, covered entities of EU ETS steadily reduce their carbon 
emission every year, and have over-fulfilled its emission reduction commitment in Kyoto Protocol. Second, EU's total cost of carbon emission reduction was reduced by $21 \%$ through allowance trading and EUR3.1 to EUR3.9 billion is saved annually, motivating enterprises to innovate in energy saving and emission reduction technologies. Third, a structured carbon finance market that includes basic and derivative products was constructed. Its transaction value has reached EUR93 billion in 2010, which preliminarily possessed the function of price signaling of carbon emission trading. Meanwhile, the European pattern is also confronted with urgent problems. The most notable ones are over surplus of allowance and slumping of market price, which are closely related and have been repeated periodically. They both occurred in the periods of year 2005 to 2007 and year 2008 to 2012 .

In the first stage, the emission reduction target of EU ETS was to accomplish $45 \%$ of its committed target in Kyoto Protocol and its annual cap of allowance was set as 2.2 billion tons of CO2e(EC,2003), where Germany occupied 25\%, UK, Poland and Italy accounted for $10 \%$ respectively, Bulgaria was $1 \%$; the proportion of emissions from electric and thermal power industries was 55\%, nonmetal and steel industries was $12 \%$ respectively, petroleum and natural gas industries was $10 \%$. Statistics shows that: in the first stage, in EU, the actual emission amount was lower than cap of allowance by $2 \%$, actual reduced emission was more than reduced allowance, and supply of allowance was slightly larger than demand for it. However, sector structure was extremely disproportionate. Output shrinking in industries like nonmetal, steel, petroleum and natural gas led to industrial allowance over surplus and became the major suppliers of allowance; whereas electric and thermal power was short of carbon allowance and became the major demanders of allowance; demand and supply within an industry was also seriously disproportionate. Over surplus and structural disproportion led to violent price fluctuation in EU carbon market and some industries and enterprises gained unreasonably excessive profits from allowance allocation[3].

In the second stage, having learned lessons from last stage and allowing for background alteration of its members, EU adjusted its carbon emission trading. First of all, cap of allowance has cut down to 2.098 billion tons of CO2-e, dropping by about $6.5 \%$ compared with the actual emission of the first stage. Second, the distribution structure of allowance was adjusted as well: the allowance and proportion of the original 15 nations of EU decreased, where Germany occupied $22 \%$, UK $12 \%$, Poland 10\%, Italy 9\%; carbon allowance of newly joined 12 nations increased, where proportion of Bulgaria and Estonia and other countries. The allowance and proportion of electric and thermal power had been decreased; proportion of allowance for aviation was 3\%, and chemistry was $3 \%$. However, EU's economy recessed because of impacts of succeeding global financial crises and EU debt crises. Severe over surplus of allowance recurred in EU ETS in the second stage. During 2008-2012, outputs of power, steel, paper-making and other sectors were vastly lower than the anticipated values, and the total amount of allowance was higher than that of actual carbon emission by 1.8 billion tons of CO2e (equaled to $20 \%$ of the actual emission). Supply of allowance greatly exceeded demand for it and market price of allowance dropt continuously[4]. Given the lessons from withdrawing allowance in the first stage, EU regulated that carbon allowance of the second stage could be restored and used in the third stage. At a time when the prospect of EU economy was still not bright, allowing huge allowance spread stored undoubtedly would result in more severe unbalance of demand and supply. The governor department of EU ETS thereby suggested backloading, namely, pulling out 0.9 billion tons of carbon allowance from the over supplied market in the form of postponed auction in order to adjust demand and supply, and to maintain present market price of allowance. However, "Backloading" was not approved by the parliament of EU because of the nature of allowance being private property, indeterminate impacts on futures market and future allowance price as well as protests from coal industry etc., resulting in another round of price slumping in EU ETS market.

\section{Theory Analysis of the EU-ETS}

First of all, why is the fixed cap always larger than actual demand for allowance in the carbon emission market of EU? Or in another word, why does the actual carbon emission reduction always exceed the carbon reduction anticipated by EU ETS? 
Starting from the hypothesis of determining the price by the equilibrium point of demand and supply of micro-economics, actual carbon emission can be viewed as the demander for allowance, and allowance price and actual demand for allowance makes up a right downward sloping demand curve. Lower carbon price means more carbon emission, vice versa. In the same way, it can be assumed that the allocated allowance is the supplier; the slope of the theoretical curve is marginal carbon emission reduction cost; where higher cost means more allowance. In an ideal situation, the intersection of curves of demand and supply is the balanced price of allowance. However, in practice, the curve of allowance supply of EU carbon emission trading market is not decided by actual supply curve of marginal carbon emission reduction cost, but by the policy targeted supply of allowance from the outsiders. This will cause the inconsistency between actual emission reduction and policy target, which is not only reflected by cap inconsistency, but also by structural displacement.

Supposed that EU could gain complete information of marginal carbon emission reduction cost of all industries, however, due to the unpredictability of economic activities, EU does not and cannot possess complete information of demand and supply of allowance in actual practice when carbon emission is the function of output. It becomes a normal state that allowance does not equal actual emission, which is represented by curve of carbon supply shifts right or left in the two dimensional flat surface of demand and supply. When it shifts right, its intersection with the curve of demand will be at the lower allowance price; when it shifts left, its intersection with the curve of demand will be at the higher allowance price. In the same way, curve of demand may shift as well, causing fluctuation of carbon emission price. Please note that: the shifting of curves of demand and supply here is caused by external economic disturbance which is irrelevant to carbon emission reduction actions. Similar to this, although EU has strictly restricted the integrated industries in carbon emission trading market, it is still a market where multiple industries trade together, which means, both curves of demand and supply are formed by the adding up of multiple industries. The curves of each industry can be shifted, which in practice is reflected by structural displacement among industries in EU carbon emission trading market in the first and second stages.

Secondly, it can be briefly proved that under the pattern of fixed cap, allowance price is not determined by marginal carbon emission reduction cost but by the difference between caps regulated by the policy and the actual emission.

Suppose $t$ is the baseline period, $t+1$ is the allocation period; $C E$ is cap, namely, total supply of allowance; $V$ is carbon intensity and $Q$ is output, $E$ is actual emission, namely actual demand for allowance, then $E=V Q$.

(1) EU ETS sets cap of allowance in light of targeted carbon intensity and anticipated output in the allocation period, where cap $C E_{t+1}=\bar{V}_{t+1} \bar{Q}_{t+1}$ and $\bar{V}_{t+1}, \bar{Q}_{t+1}$ are respectively targeted carbon intensity and anticipated output during the allocation period.

(2) The actual emission in the baseline period $E_{t}=V_{t} Q_{t}$; the actual emission in allocation period is $E_{t+1}=V_{t+1} Q_{t+1}$;

(3) Cap Reduction, $C R_{t+1}=E_{t}-C E_{t+1}=V_{t} Q_{t}-\bar{V}_{t+1} \bar{Q}_{t+1}$

(4) Real Reduction, $R R_{t+1}=E_{t}-E_{t+1}=C R_{t+1}+\left(C E_{t+1}-E_{t+1}\right)$

(5) Difference between Real Reduction and Cap Reduction, $W_{t+1}$ :

$$
W_{t+1}=R R_{t+1}-C R_{t+1}=\bar{V}_{t+1}\left(\bar{Q}_{t+1}-Q_{t+1}\right)+\left(\bar{V}_{t+1}-V_{t+1}\right) Q_{t+1}
$$

Cap setting in carbon emission trading aims at maximization of reduction efficacy, which can be expressed by $\max U\left(R R_{t+1}\right)=\max U\left(C R_{t+1}+W_{t+1}\right)$, only and if only $W_{t+1} \rightarrow 0$, i.e., no variance between anticipated output and technical level, $R R_{t+1}=C R_{t+1}$, supply of allowance may equal actual demand for it. However, in practice, $\bar{Q}_{t+1}-Q_{t+1} \in N(0,1), \bar{V}_{t+1}-V_{t+1} \in N(0,1)$ generally complies with white noise distribution, therefore $\mathrm{Et}+1$ is not always equal to $\mathrm{CEt}+1$, Cap Reduction is not always equal to Real Reduction, either. Based on $\bar{V}_{t+1}, \bar{Q}_{t+1}$ Cap setting and allocation in different sectors will be at the risk of random adjustment. In the first two stages of EU-ETS, $\bar{Q}_{t+1}>Q_{t+1}$, variant output 
fluctuation of integrated industries is the main reason for over surplus of allowance and disproportionate allocation among sectors.

(6) Effective Reduction of carbon intensity, i.e., ERt+1:

$E R_{t+1}=\left(V_{t}-V_{t+1}\right) Q_{t+1}$

$E R_{t+1}=C R_{t+1}+V_{t}\left(Q_{t+1}-Q_{t}\right)+\left(C E_{t+1}-E_{t+1}\right)$

$E R_{t+1}=R R_{t+1}+V_{t}\left(Q_{t+1}-Q_{t}\right)$

In the pattern of EU, cap reduction is an observable policy target, while actual output and anticipated output, variance between actual emission and allowance cap need to be analyzed later. Therein, output and carbon intensity jointly influence the emission change of greenhouse gases, therefore to clarify their degree of contribution and influence is the technical foundation of attaining reasonable carbon price. As price is determined by demand and supply in market, the equilibrium point of demand and supply is the point of tangency of marginal rate of element substitution rate and marginal level of output, then allowance price $P_{c, t+1}=M C_{t+1}\left(E R_{t+1}\right)$. If the actual output $Q_{t+1}=\bar{Q}_{t+1}$, then:

$$
P_{c, t+1}=M C_{t+1}\left(E R_{t+1}\right)=M C_{t+1}\left(R R_{t+1}\right)=M C_{t+1}\left(C R_{t+1}\right)
$$

When the output is unstable, the real reduction is not equal to actual reduction or allowance reduction. If cap does not alter, supply of allowance is always equal to $\mathrm{CE}_{\mathrm{t}+1}$, demand for allowance is $V_{t+1} Q_{t+1}$, then the theoretical equilibrium price becomes $M C\left(V_{t+1} Q_{t+1}-C E_{t+1}\right)=M C\left(E_{t+1}-C E_{t+1}\right)$. It is easy to infer that in the mechanism of fixed cap, because of the instability of output, carbon price is not relevant to marginal carbon emission reduction cost, but is the function of actual emission and fixed reduction targets or carbon allowance allocation. The fluctuation of price is determined by the degree of over surplus of cap.

\section{Conclusions}

The experiences of and the lessons from EU carbon emission trading indicate that: in the pattern of the EU ETS, cap and its allocation are determined by the expected output, measuring of industrial emission reduction potential, as well as the negotiation skills between the government and enterprises. Judging from its performance in the first two periods, the mechanism of carbon allowance allocation should be improved and introduces some innovative methods.

\section{Acknowledgements}

This work was supported by The Natural Science Foundation of GuangDong Province, China (Grant No. 2014A030310404).

\section{References}

[1] Jiang J J, Ye B. Ma X M. The Construction of Shenzhen's Carbon Emission Trading Scheme [J]. Energy Policy, 2014, 75: 17-21.

[2] Ye B, Jiang J. Miao L, et al. Sustainable Energy Options for a Low Carbon Demonstration City Project in Shenzhen, China[J]. Journal of Renewable and Sustainable Energy, 2015, 7(2): 2311723122.

[3] Ellerman A., A note tradable permits [J]. Environmental and Resource economics, 2005, 31: 123131.

[4] Jie T, Bin Y. Qiang L, et al. Economic Analysis of Photovoltaic Electricity Supply for an Electric Vehicle Fleet in Shenzhen, China[J]. International Journal of Sustainable Transportation, 2014, 8(3):202-224. 